\title{
A ROBUST PREDICTIVE CONTROL DESIGN FOR NONLINEAR ACTIVE SUSPENSION SYSTEMS
}

\author{
Sofiane Bououden, Mohammed Chadli, and Hamid Reza Karimi
}

\begin{abstract}
This paper proposes a novel method for designing robust nonlinear multivariable predictive control for nonlinear active suspension systems via the Takagi-Sugeno fuzzy approach. The controller design is converted to a convex optimization problem with linear matrix inequality constraints. The stability of the control system is achieved by the use of terminal constraints, in particular the Constrained Receding-Horizon Predictive Control algorithm to maintain a robust performance of vehicle systems. A quarter-car model with active suspension system is considered in this paper and a numerical example is employed to illustrate the effectiveness of the proposed approach. The obtained results are compared with those achieved with model predictive control in terms of robustness and stability.
\end{abstract}

Key Words: Active vehicle suspension, fuzzy models, model predictive control, linear matrix inequality, optimization.

\section{INTRODUCTION}

In general, vehicle suspension systems are used to create ride comfort by isolating the chassis mass from road disturbances and to improve road holding by preventing the wheel from losing road contact. Many systems have been developed to improve the road-holding ability and ride quality of cars, as indicated in the literature review on suspension systems.

Generally speaking, suspension systems can be categorized into passive suspensions, semi-active suspensions, and active suspension systems [1-3], which have been developed for achieving the required vehicle performance. The active suspension system is more elastic and efficient than other suspension systems, making it more able to offer road-holding ability and ride quality. Therefore, active suspension systems control has attracted the attention of numerous researchers interested in ride and holding qualities. However, active vehicle suspension system models assume a small displacement about an operating point, and then it creates a linearized working model [4-6]. On the other hand, the system states exhibit large deviation from the equilibrium point when the system is subjected to major impact owing to rough roads or aggressive driving.

The performance obtained by these conventional controllers cannot be regarded as satisfactory. Therefore,

S. Bououden is with the Faculty of Sciences and Technology, University of Abbes Laghrour Khenchela, Algeria (e-mail: ss.bououden@gmail.com).

M. Chadli is with the University of Picardie Jules Verne, MIS (EA 4029), 33 rue Saint-Leu, 80039 Amiens, France (e-mail: mchadli@u-picardie.fr).

H. R. Karimi is with the Department of Engineering, Faculty of Engineering and Science, University of Agder, Grimstad 4898, Norway (e-mail: hamid.r.karimi@uia.no). improvement in performance is expected if the nonlinearities of the system can be included in the control design. While such a treatment can be complicated when implementing classical continuous optimal control, stability analysis and controller synthesis theory for nonlinear systems have received much attention recently. Different nonlinear active suspensions based on various control techniques include: the sliding model controller [7], fuzzy logic and neural network control [8], adaptive control [9], and nonlinear control [10], which are based on the idea of minimizing a single objective function without any constraints.

Nonlinear Predictive Control (NPC) in conjunction with hybrid modeling can be an attractive and systematic methodology to address these challenging control problems. Owing to its ability to handle the constrained optimization control problems for multiple-input multiple-output (MIMO) systems, and with its disturbance rejection properties, model predictive control (MPC) appears to be an appropriate technique [11-14]. On the other hand, while the predictive control approaches take into account the actuator constraints, they are currently limited since the required measurements do not cope with the industrial costs and reliability objectives.

This paper describes a new control strategy for the active car suspension system. A robust nonlinear multivariable predictive control (RNMPC) under constraints is proposed and solved using a linear matrix inequalities (LMIs) formulation. This new approach is suitable, as both performance and robustness issues are handled within a unified framework. Firstly, the Takagi-Sugeno (T-S) fuzzy model is used to approximate the nonlinear active vehicle suspension systems [15-18]. Then, an NPC is built, and reformulated as an optimization problem of robust quadratic programming. Using LMIs, a design stability condition is 
introduced, which forces all possible changes to the system state to converge on a finite horizon, or to reach a terminal area for which a robust control law stabilizes the system. Based on this approach, the suspension system is optimized with respect to sprung mass acceleration, suspension deflection, and tire deflection

This paper is structured as follows. In Section II, the description of a class of active suspension systems and its representation by a T-S fuzzy model are proposed. Section III presents the formulation of a predictive control problem for a quarter-car model. In Section IV, a robust predictive control subject to LMI constraints is given. Simulation results are presented in Section V. Section VI concludes the paper.

\section{MODELING OF NONLINEAR ACTIVE SUSPENSION SYSTEM}

\subsection{Active quarter-car suspension}

The quarter car model illustrated in Fig. 1 is considered here for designing active suspension control laws. This model is a two-DOF (degree of freedom) quarter-car model and considers both the vehicle sprung mass and the unsprung mass associated with the wheel/tire/axle assembly.

The dynamic equations that govern the motions of the sprung and unsprung masses are represented as follows

$$
\begin{aligned}
m_{s} \ddot{z}_{s}(t) & +c_{s}\left(\dot{z}_{s}(t)-\dot{z}_{u s}(t)\right)+k_{s}\left(z_{s}(t)-z_{u s}(t)\right)=-u(t) \\
m_{u s} \ddot{z}_{u s}(t) & +c_{s}\left(\dot{z}_{u s}(t)-\dot{z}_{s}(t)\right)+k_{s}\left(z_{u s}(t)-z_{s}(t)\right) \\
& +c_{u s}\left(\dot{z}_{u s}(t)-\dot{z}_{o}(t)\right)+k_{u s}\left(z_{u s}(t)-z_{o}(t)\right)=u(t)
\end{aligned}
$$

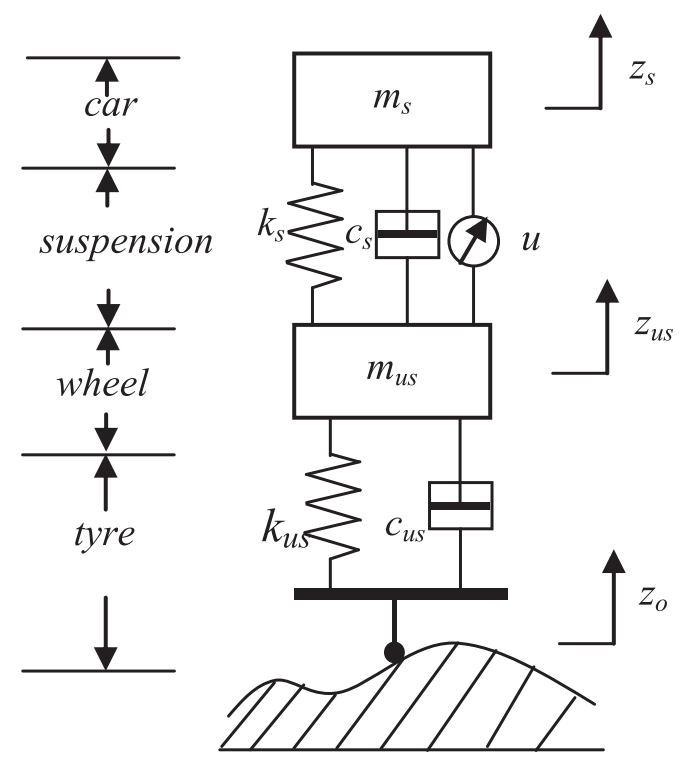

Fig. 1. The active quarter-car suspension system. where $z_{s}(t)$ and $z_{u s}(t)$ represent the vertical displacements of the sprung mass and unsprung mass, respectively; $z_{o}(t)$ is the road displacement input; $u(t)$ represents the active control force of the suspension system; $m_{s}$ denotes the sprung mass, which represents the car chassis; $m_{u s}$ is the unsprung mass, which represents the mass of the wheel assembly; $k_{s}$ and $c_{s}$ are spring coefficient of suspension system and damping coefficient of suspension system, respectively; $k_{u s}$ and $c_{u s}$ stand for compressibility and damping of the pneumatic tire, respectively.

The equations of motion in standard-state variable form are as follows

$$
\begin{aligned}
& \frac{d}{d t}\left[\begin{array}{c}
z_{u s}(t)-z_{0}(t) \\
\dot{z}_{u s}(t) \\
z_{s}(t)-z_{u s}(t) \\
\dot{z}_{s}(t)
\end{array}\right]=\left[\begin{array}{cccc}
0 & 1 & 0 & 0 \\
-\frac{k_{u s}}{m_{u s}} & \frac{\left(c_{s}+c_{u s}\right)}{m_{u s}} & \frac{k_{s}}{m_{u s}} & \frac{c_{s}}{m_{u s}} \\
0 & -1 & 0 & 1 \\
0 & \frac{c_{s}}{m_{s}} & -\frac{k_{s}}{m_{s}} & -\frac{c_{s}}{m_{s}}
\end{array}\right] \\
& \times\left[\begin{array}{c}
z_{u s}(t)-z_{0}(t) \\
\dot{z}_{u s}(t) \\
z_{s}(t)-z_{u s}(t) \\
\dot{z}_{s}(t)
\end{array}\right]+\left[\begin{array}{cc}
0 & -1 \\
\frac{1}{m_{u s}} & \frac{c_{u s}}{m_{u s}} \\
0 & 0 \\
-\frac{1}{m_{s}} & 0
\end{array}\right]\left[\begin{array}{c}
u(t) \\
\dot{z}_{0}(t)
\end{array}\right]
\end{aligned}
$$

which can be further expressed by

$$
\dot{x}(t)=A x(t)+B w(t)
$$

where the state variable are the tire deflection $x_{1}(t)=$ $z_{u s}(t)-z_{0}(t)$, the unsprung-mass velocity $x_{2}(t)=\dot{z}_{u s}(t)$, the suspension stroke $x_{3}(t)=z_{s}(t)-z_{u s}(t)$, and the sprung-mass velocity $x_{4}(t)=\dot{z}_{s}(t)$. The coefficients of the state equation also can be presented in the following form

$$
\begin{aligned}
& A=\left[\begin{array}{cccc}
0 & 1 & 0 & 0 \\
-\frac{k_{u s}}{m_{u s}} & \frac{\left(c_{s}+c_{u s}\right)}{m_{u s}} & \frac{k_{s}}{m_{u s}} & \frac{c_{s}}{m_{u s}} \\
0 & -1 & 0 & 1 \\
0 & \frac{c_{s}}{m_{s}} & -\frac{k_{s}}{m_{s}} & -\frac{c_{s}}{m_{s}}
\end{array}\right], \\
& B=\left[\begin{array}{cc}
0 & -1 \\
\frac{1}{m_{u s}} & \frac{c_{u s}}{m_{u s}} \\
0 & 0 \\
-\frac{1}{m_{s}} & 0
\end{array}\right]
\end{aligned}
$$

In (1) the springs and dampers of suspension systems are usually modeled as linear elements. To show the effect of dropping the nonlinear terms, assume a nonlinear model 
where only primary spring nonlinearities appear. Therefore, the following nonlinear differential equations are obtained for the active car suspension system

$$
\begin{aligned}
m_{s} \ddot{z}_{s}(t) & +c_{s}\left(\dot{z}_{s}(t)-\dot{z}_{u s}(t)\right)+k_{s}\left(z_{s}(t)-z_{u s}(t)\right) \\
& +N_{s}\left(z_{s}(t)-z_{u s}(t)\right)^{3}=-u(t) \\
m_{u s} \ddot{z}_{u s}(t) & +c_{s}\left(\dot{z}_{u s}(t)-\dot{z}_{s}(t)\right)+k_{s}\left(z_{u s}(t)-z_{s}(t)\right) \\
& +N_{s}\left(z_{s}(t)-z_{u s}(t)\right)^{3}+c_{u s}\left(\dot{z}_{u s}(t)-\dot{z}_{o}(t)\right) \\
& +k_{u s}\left(z_{u s}(t)-z_{o}(t)\right)=u(t)
\end{aligned}
$$

where $N_{s}$ is the nonlinear stiffness property of the primary suspension.

The design procedure begins with representing a given nonlinear plant by T-S fuzzy model [19-21].

In the next section, the T-S fuzzy system is used to describe the original nonlinear system for the control-design aim via the sector nonlinearity approach.

\subsection{T-S fuzzy representation of suspension system}

T-S fuzzy systems provide an effective representation of uncertain nonlinear systems described by a set of linear models. To obtain a T-S fuzzy model it is just enough that the decision variables are assumed varying in the operating range $-\beta \leq x_{3}(t) \leq \beta$. Then the T-S fuzzy model of vehicle suspension control system can be described by the following two IF-THEN rules [22]

If $x_{2}$ is $F_{1}^{1}$ Then

$$
\left\{\begin{array}{l}
\dot{x}(t)=\left(A_{1}+\delta A_{1}\right) x(t)+\left(B_{1}+\delta B_{1}\right) w(t) \\
y(t)=(C+\delta C) x(t)
\end{array}\right.
$$

If $x_{2}$ is $F_{1}^{2}$ Then

$$
\left\{\begin{array}{l}
\dot{x}(t)=\left(A_{2}+\delta A_{2}\right) x(t)+\left(B_{2}+\delta B_{2}\right) w(t) \\
y(t)=(C+\delta C) x(t)
\end{array}\right.
$$

The active vehicle suspension can be represented by the following T-S fuzzy model

$$
\left\{\begin{array}{l}
\dot{x}(t)=\sum_{i=1}^{4} h_{i}\left(\left(A_{i}+\delta A_{i}\right) x+\left(B_{i}+\delta B_{i}\right) w(t)\right) \\
y=(C+\delta C) x
\end{array}\right.
$$

In this work we consider the model of active quartercar suspension with nonlinearity effect of suspension given by (4), and let the variable of premise is defined as

$$
z(t)=\left(x_{3}(t)\right)^{2}
$$

when, $x_{3}(t)=[-\beta, \beta]$, and $z(t)=\left[0, \beta^{2}\right], z(t)=\beta F_{1}^{1}\left(x_{3}(t)\right)-$ $\beta F_{1}^{2}\left(x_{3}(t)\right)$, with

$$
\begin{aligned}
& h_{1}(z)=F_{1}^{1}\left(x_{3}(t)\right), h_{2}(z)=F_{1}^{2}\left(x_{3}(t)\right) \\
& F_{1}^{1}\left(x_{3}(t)\right)=\frac{x_{3}(t)+\beta}{2 \beta}, F_{1}^{2}\left(x_{2}\right)=1-F_{1}^{1}\left(x_{3}(t)\right)
\end{aligned}
$$

The nonlinear system (4) can be exactly represented in the compact set $[-\beta, \beta]$ of the variable $x_{3}(t)$ by the following state-space matrices of the two rules in the T-S fuzzy model

$$
\begin{aligned}
& A_{1}=\left[\begin{array}{cccc}
0 & 1 & 0 & 0 \\
-\frac{k_{u s}}{m_{u s}} & \frac{\left(c_{s}+c_{u s}\right)}{m_{u s}} & \frac{k_{s}}{m_{u s}} & \frac{c_{s}}{m_{u s}} \\
0 & -1 & 0 & 1 \\
0 & \frac{c_{s}}{m_{s}} & \frac{-k_{s}}{m_{s}} & -\frac{c_{s}}{m_{s}}
\end{array}\right], \\
& A_{2}= {\left[\begin{array}{cccc}
0 & 1 & 0 & 0 \\
-\frac{k_{u s}}{m_{u s}} & \frac{\left(c_{s}+c_{u s}\right)}{m_{u s}} & \frac{\left(k_{s}-N_{s} \beta\right)}{m_{u s}} & \frac{c_{s}}{m_{u s}} \\
0 & -1 & 0 & 1 \\
0 & \frac{c_{s}}{m_{s}} & -\frac{\left(k_{s}+N_{s} \beta\right)}{m_{s}} & -\frac{c_{s}}{m_{s}}
\end{array}\right] } \\
& B_{1}=B_{2}=\left[\begin{array}{cc}
0 & -1 \\
\frac{1}{m_{u s}} & \frac{c_{u s}}{m_{u s}} \\
0 & 0 \\
-\frac{1}{m_{s}} & 0
\end{array}\right],
\end{aligned}
$$

Therefore the entire T-S car suspension model corresponds to

$\left\{\begin{array}{l}x(k+1)=(A(k)+\delta A(k)) x(k)+(B(k)+\delta B(k)) w(k) \\ y(k)=(C(k)+\delta C(k)) x(k)\end{array}\right.$

where $A(k)=\sum_{i=1}^{r} h_{i}(z(k)) A_{i}, B(k)=\sum_{i=1}^{r} h_{i}(z(k)) B_{i}, C(k)=$ $\sum_{i=1}^{r} h_{i}(z(k)) C_{i}$

$$
\delta A(k)=\sum_{i=1}^{r} h_{i}(z(k)) \delta A_{i}, \delta B(k)=\sum_{i=1}^{r} h_{i}(z(k)) \delta B_{i},
$$

In the next section, the control strategy, which takes into account constraints such as control effort and state variables, is presented.

\section{MODEL PREDICTIVE CONTROLLER DESIGN}

The main advantages of MPC include the ability to handle constraints and the capability for controlling multivariable plants. This section presents details of the application of MPC based on the mixed control approach for active 
suspension vehicle control. The MPC approach utilizes (6) as the internal model of the actuator dynamics to predict $y(t)$ at a future discrete time instants $\left[\hat{y}\left(k+H_{1} / k\right), \ldots\right.$, $\left.\hat{y}\left(k+H_{p} / k\right)\right]$. In this representation $\hat{y}(k+j / k)$ denotes the optimal $j$-step ahead prediction of the system output, based on data up to time $k$, while $H_{1}$ and $H_{\mathrm{p}}$ are respectively the lower and upper limits of the receding horizon; the predictive controller computes an optimal control sequence with respect to the following cost function [23-25]

$$
\begin{aligned}
\min _{u} J= & \sum_{j=H_{1}}^{H_{p}}(r(k+j)-\hat{y}(k+j / k))^{T} Q(r(k+j)-\hat{y}(k+j / k)) \\
& +\sum_{j=1}^{H_{u}}\left(u(k+j-1)^{T} R u(k+j-1)\right. \\
& \left.+\Delta u(k+j-1)^{T} S \Delta u(k+j-1)\right)
\end{aligned}
$$

where $H_{u}$ is the control horizon, $Q$ is the weighting matrix of the tracking error, $R$ and $S$ are weighting matrices of the control effort, $r(k)$ is the reference trajectory vector, $u(k)$ is the control command and $\delta u(k)$ is the incremental control command representing the energy needed to actuate the control effector. The control objective $u(k), u(k+1) \ldots$ is used to achieve good ride quality while maintaining adequate suspension clearance, i.e. minimizing the acceleration of the vehicle body experienced by passengers without causing large suspension deflections.

Actuator displacement and the actuator displacement rate are mechanically limited and these constraints are incorporated. Mathematically (7) can be expressed in a matrix form as [26]

$$
\begin{aligned}
J\left(U_{n}\right)= & J_{\min }+2\left[\left(\Gamma-Y_{r e f}^{n}\right)^{T} Q \Lambda-\bar{U}_{k-1}^{T} S \Delta\right] U_{n} \\
& +U_{n}^{T}\left[\Lambda^{T} Q \Lambda+R+\Delta^{T} S \Delta\right] U_{n}
\end{aligned}
$$

where

$$
J_{\min }=Y_{r e f}^{T} Q Y_{r e f}+\Gamma^{T} Q \Gamma-2 Y_{r e f}^{T} Q \Gamma+\bar{U}_{k-1}^{T} S \bar{U}_{k-1}^{T}
$$

Subject to

$$
\left[\begin{array}{l}
L_{H_{u}+1} \\
-L_{H_{u}+1} \\
I_{H_{u}+1} \\
-I_{H_{u}+1} \\
\Lambda \\
-\Lambda
\end{array}\right] U_{n} \leq\left[\begin{array}{l}
U_{\max }+U_{k-1} \\
-U_{\min }-U_{k-1} \\
U_{\max } \\
-U_{\min } \\
Y_{\max }-\Gamma \\
-Y_{\max }+\Gamma
\end{array}\right]
$$

Where the predicted output is given by (see Appendix VII)

$$
\hat{Y}=\Gamma+\Lambda U_{n}
$$

and $\hat{Y} \in R^{H_{p} n_{0}}, \Gamma \in R^{H_{p} n_{0}}, \Lambda \in R^{H_{p} n_{0} \times H_{u} n_{i}}$ and $\Delta U_{n} \in R^{H_{u} n_{i}}, n_{0}$ and $n_{i}$ are the number of system outputs and system inputs, respectively. $\Gamma$ is called the free output response as it does not depend on the control sequence and it is obtained by simulation of the free response in the nonlinear model; and $\Lambda \Delta U_{n}$ is called the force output response as it depends on the selected control sequence where $\Lambda$ is result of a linearization.

\subsection{CRHPC}

The simplest method of enforcing stability with a finite prediction horizon is to add a so-called terminal equality constraint at the end of the prediction horizon [27,28], i.e. to add the equality constraint to the optimization problem (8) and (9). To guarantee the stability of the system, the following set of terminal equality constraints is included in the optimization problem

$$
y\left(t+H_{p}+j\right)=Y_{r e f}\left(t+H_{p}\right), j=1, \cdots, m
$$

where $m$ is instants beyond the prediction horizon, representing the terminal constraint.

The prediction that is extended for $m$ instants beyond the prediction horizon results in (c index signify the terminal constraint) (see Appendix VIII)

$$
\hat{Y}_{c}=\Gamma_{c}+\Lambda_{c} U_{n},
$$

where $\Gamma_{c}$ is the free response prediction extended for $m$ instants beyond the prediction horizon and $\Lambda_{c} U_{n}$ is the forced response prediction, based on future control actions.

Substituting (12) into (11), the terminal constraints (11) can be reformulated in terms of optimization an upper bound, then writing in a consistent form with the LMI structure: (see Appendix VIII)

$$
\begin{aligned}
& \Lambda c U_{n}-\left(Y_{\text {ref }}-\Gamma_{c}\right)-\alpha \gamma I_{m}<0 \\
& -\Lambda c U_{n}+\left(Y_{r e f}-\Gamma_{c}\right)-\alpha \gamma I_{m}<0
\end{aligned}
$$

In practice, $\alpha<<1$ proves to be sufficient to ensure the equivalence between (13) and (11).

\subsection{Robust Fuzzy Predictive Control}

Owing to the disturbances caused by road bumpiness, firm, uninterrupted contact of wheels with road (good road holding) is important for vehicle handling and is essentially related to driving safety. Hence, it is also important to introduce disturbances for solving the optimization problem. In this work we modify the minimization of the nominal objective function (8) to a minimization of the worst-case objective function.

By introducing uncertainty in the parameters of the system (6), the predicted output will be restricted to the forced response term $(\Lambda+\delta \Lambda) U$, and the output prediction with the terminal constraint is as follows 


$$
\begin{aligned}
& \hat{Y}=\Gamma+\Lambda U_{n} \\
& \hat{Y}_{c}=\Gamma_{c}+\Lambda_{c} U_{n}
\end{aligned}
$$

Replacing (10) and (12) with (14) and (15), the cost function (8), constraints (9), and CRHPC (12) can be converted as

$$
\begin{aligned}
J\left(U_{n}\right)= & J_{\min }+2\left[\left(\Gamma-Y_{r e f}^{n}\right)^{T} Q \Lambda-\bar{U}_{k-1}^{T} S \Delta\right. \\
& \left.+\left(\Gamma-Y_{r e f}^{n}\right)^{T} Q \delta \Lambda\right] U_{n}+U_{n}^{T}\left[\Lambda^{T} Q \Lambda+R\right. \\
& \left.+\Delta^{T} S \Delta+\delta \Lambda^{T} Q \delta \Lambda+\Lambda^{T} Q \delta \Lambda+\delta \Lambda^{T} Q \Lambda\right] U_{n}
\end{aligned}
$$

Subject to

$$
\left[\begin{array}{l}
L_{H_{u}+1} \\
-L_{H_{u}+1} \\
I_{H_{u}+1} \\
-I_{H_{u}+1} \\
(\Lambda+\delta \Lambda) \\
-(\Lambda+\delta \Lambda) \\
\left(\Lambda_{c}+\delta \Lambda_{c}\right) \\
-\left(\Lambda_{c}+\delta \Lambda_{c}\right)
\end{array}\right] U_{n}<\left[\begin{array}{l}
U_{\max }+U_{k-1} \\
-U_{\min }-U_{k-1} \\
U_{\max } \\
-U_{\min } \\
Y_{\max }-\Gamma \\
-Y_{\min }+\Gamma \\
Y_{r e f}-\Gamma_{c}+\alpha I_{m} \\
-Y_{r e f}+\Gamma_{c}+\alpha I_{m}
\end{array}\right]
$$

Observe that no uncertainty is considered in the free responses $\Gamma$ and $\Gamma_{c}$.

The following section illustrates the problem formulations, i.e. model for uncertain systems, modification of the cost function by deriving an upper bound, and the motivation of the LMI approach. Note that all the constraints should be transferred in terms of LMI constraints.

\subsection{Formulation of the predictive control problem as a quadratic program}

Optimization problems associated with predictive control belong to the class of convex optimization problems since the cost function (16) and feasible set (17) are convex [29]. So our problem consists of minimizing a convex function (16) on a convex domain (17). This convex minimization has a global minimum only (if any) if the Hessian of the function objective is positive definite [30,31]. In this sense, the MPC objective function, (16), can be transformed into the form

$$
\min U_{n}(k)^{T} H U_{n}(k)-P^{T} U_{n}(k)+E
$$

Subject to the inequality constraints (15),

where the Hessien matrix $H$ is positive definite if the matrix $\Lambda$ satisfies

$$
\operatorname{rank}(\Lambda)=H_{u}
$$

we see from (14) that the constraints may be structured as a single formalism easily exploitable later by the optimization algorithms

$$
A U_{n}(k) \leq B
$$

Criterion (18) is nonlinear in terms of quadratic terms, and for its transformation into LMI form, we will use the Schur complement lemma. This formalism can minimize linear cost function with LMI constraints [32]

$$
\begin{aligned}
& \min c^{T} x \\
& \text { Subject to } F(x)<0
\end{aligned}
$$

where $F$ is a symmetric matrix that depends affinely on the optimization variable $x$, and $c$ is a real vector of appropriate size. The solution is then to minimize the linear term $\left\langle c^{T} x\right\rangle$. This is the observation about LMI-based optimization that is most relevant to us. The next section uses the LMI formalism as an optimization tool.

\section{LMI APPROACH FOR ROBUST QUADRATIC PROGRAM}

The main advantage of the LMI formulations is the ability to combine various design constraints so that the robust fuzzy control design based on LMIs can be readily solved. In summary, the principle of this approach is to minimize an upper bound of the objective function with restrictions on the input and output. It transfers the uncertainties from the cost function to the constraints in order to simplify the online computational complexity.

In general, the minimization of a convex quadratic function $J\left(U_{n}\right)$ can result in an equivalent manner by the strategy of minimizing.

Minimize $\gamma$ and find an admissible $U_{n}$ satisfying

$$
J\left(U_{n}\right)<\gamma
$$

Convex quadratic inequality (23) is converted to LMI form using the Schur complements [32]. Let $L(x)=L(x)^{T}$, $M(x)=M(x)^{T}$ and $W(x)$ depend affinely on $x$. Then the following LMIs are equivalent

i) $\quad\left[\begin{array}{cc}L(x) & W(x) \\ W(x)^{T} & M(x)\end{array}\right]<0$

ii)

$$
\left\{\begin{array}{l}
M(x)<0 \\
L(x)-W(x) M(x)^{-1} W(x)^{T}<0
\end{array}\right.
$$

Inequality (22) is strict but nonlinear. From the Schur complement, the original optimization problem can be formulated as

Min $\gamma \in R^{+}$

Subject to 


$$
\begin{aligned}
& {\left[\begin{array}{l}
2\left[\left(\Gamma-Y_{r e f}^{n}\right)^{T} Q \Lambda-\bar{U}_{k-1}^{T} S \Delta+\left(\Gamma-Y_{r e f}^{n}\right)^{T} Q \delta \Lambda\right] U_{n}+J_{\min }-\gamma \\
U_{n}
\end{array}\right.} \\
& \begin{array}{l}
U_{n}^{T} \\
\left.-\left[\Lambda^{T} Q \Lambda+R+\Delta^{T} S \Delta+\delta \Lambda^{T} Q \delta \Lambda+\Lambda^{T} Q \delta \Lambda+\delta \Lambda^{T} Q \Lambda\right]^{-1}\right]<0
\end{array} \\
& -\left[\Lambda^{T} Q \Lambda+R+\Delta^{T} S \Delta\right]^{-1}<0
\end{aligned}
$$

sampling time of $T=0.5 \mathrm{~ms}$. In the simulation the parameters are set as follows

$$
m_{\mathrm{s}}=240[\mathrm{~kg}], \quad m_{\mathrm{us}}=36[\mathrm{~kg}], \quad k_{\mathrm{s}}=16000[\mathrm{~N} / \mathrm{m}],
$$
$k_{\mathrm{us}}=160000[\mathrm{~N} / \mathrm{m}], c_{\mathrm{s}}=980\left[\mathrm{~N}^{*} \mathrm{~s} / \mathrm{m}\right], c_{\mathrm{us}}=400\left[41 \%\right.$ of $\left.c_{\mathrm{s}}\right]$ and $N \mathrm{~s}=1600\left[10 \%\right.$ of $\left.k_{\mathrm{s}}\right]$.

Using sector nonlinearity, the discrete T-S fuzzy model of the above system is obtained. The T-S fuzzy model is

$$
\left\{\begin{array}{l}
L\left(U_{n}\right)=2\left[\left(\Gamma-Y_{r e f}^{n}\right)^{T} Q \Lambda-\bar{U}_{k-1}^{T} S \Delta+\left(\Gamma-Y_{r e f}^{n}\right)^{T} Q \delta \Lambda\right] U_{n}+J_{\min }-\gamma \\
M\left(U_{n}\right)=-\left[\Lambda^{T} Q \Lambda+R+\Delta^{T} S \Delta+\delta \Lambda^{T} Q \delta \Lambda+\Lambda^{T} Q \delta \Lambda+\delta \Lambda^{T} Q \Lambda\right]^{-1} \\
W\left(U_{n}\right)=U_{n}^{T}
\end{array}\right.
$$

$L\left(U_{n}\right), M\left(U_{n}\right)$ and $W\left(U_{n}\right)$ are the Schur matrices.

Finally, the second inequality of (26) is always verified by the Hessian structure in the MPC case. The previous constraints (17) must be presented in a diagonal form thus defining a convex and symmetric matrix space. At the end of the three previous steps, the original problem can be formulated as an LMI-based optimization problem

$$
\text { Min } \gamma \in R^{+}
$$

Subject to LMI constraints

$$
\begin{aligned}
& {\left[2\left[\left(\Gamma-Y_{r e f}^{n}\right)^{T} Q \Lambda-\bar{U}_{k-1}^{T} S \Delta+\left(\Gamma-Y_{r e f}^{n}\right)^{T} Q \delta \Lambda\right] U_{n}+J_{\min }-\gamma\right.} \\
& U_{n} \\
& U_{n}^{T} \\
& \left.-\left[\Lambda^{T} Q \Lambda+R+\Delta^{T} S \Delta+\delta \Lambda^{T} Q \delta \Lambda+\Lambda^{T} Q \delta \Lambda+\delta \Lambda^{T} Q \Lambda\right]^{-1}\right]<0 \\
& \operatorname{diag}\left(L_{H_{u}+1} U_{n}-\left(U_{\max }+U_{k-1}\right)\right) \leq 0 \\
& \operatorname{diag}\left(-L_{H_{u}+1} U_{n}+\left(U_{\min }+U_{k-1}\right)\right) \leq 0 \\
& \operatorname{diag}\left(I_{H_{u}+1} U_{n}-U_{\max }\right) \leq 0 \\
& \operatorname{diag}\left(-I_{H_{u}+1} U_{n}+U_{\min }\right) \leq 0 \\
& \operatorname{diag}\left((\Lambda+\delta \Lambda) U_{n}-Y_{\max }+\Gamma\right) \leq 0 \\
& \operatorname{diag}\left(-(\Lambda+\delta \Lambda) U_{n}+Y_{\min }-\Gamma\right) \leq 0 \\
& \operatorname{diag}\left((\Lambda c+\delta \Lambda c) U_{n}-\left(Y_{r e f}-\Gamma_{c}\right)-\alpha \gamma I_{m}\right) \leq 0 \\
& \operatorname{diag}\left(-(\Lambda c++\delta \Lambda c) U_{n}+\left(Y_{r e f}-\Gamma_{c}\right)-\alpha \gamma I_{m}\right) \leq 0
\end{aligned}
$$

\section{SIMULATION RESULTS}

In this simulation, we consider the discrete-time model of an active car suspension as given by (4), with a

$$
\begin{aligned}
A_{1} & =\left[\begin{array}{cccc}
0 & 1 & 0 & 0 \\
-\frac{k_{u s}}{m_{u s}} & \frac{\left(c_{s}+c_{u s}\right)}{m_{u s}} & \frac{k_{s}}{m_{u s}} & \frac{c_{s}}{m_{u s}} \\
0 & -1 & 0 & 1 \\
0 & \frac{c_{s}}{m_{s}} & \frac{-k_{s}}{m_{s}} & -\frac{c_{s}}{m_{s}}
\end{array}\right], \\
A_{2} & =\left[\begin{array}{cccc}
0 & 1 & 0 & 0 \\
-\frac{k_{u s}}{m_{u s}} & \frac{\left(c_{s}+c_{u s}\right)}{m_{u s}} & \frac{k_{s}-N_{s} \beta^{2}}{m_{u s}} & \frac{c_{s}}{m_{u s}} \\
0 & -1 & 0 & 1 \\
0 & \frac{c_{s}}{m_{s}} & -\frac{k_{s}+N_{s} \beta^{2}}{m_{s}} & -\frac{c_{s}}{m_{s}}
\end{array}\right] \\
B_{1}=B_{2}= & {\left[\begin{array}{cc}
0 & -1 \\
\frac{1}{m_{u s}} & \frac{c_{u s}}{m_{u s}} \\
0 & 0 \\
-\frac{1}{m_{s}} & 0
\end{array}\right] \text { with } \beta=0.2 . }
\end{aligned}
$$

We can easily obtain the discrete-time local models of fuzzy system (3) for the active suspension system (1) under the sampling time $T=0.5 \mathrm{~ms}$. The MPC parameters are selected as follows. The control horizon is $H_{u}=1$, and the prediction horizon is set only to $H_{p}=12$. The weighting matrices are $Q=\boldsymbol{I}, R=0.4 \boldsymbol{I}$ and $S=0.15 \boldsymbol{I}$. To limit the power of the hydraulic actuator, the hard constraint on the active suspension is imposed $|u(k)| \leq u_{\max } ; u_{\max }=1500$; and the CRHPC are $m=2$.

The RNPC-based LMI seen in Sections 3.2 and 3.4, shows that when calculating the objective function, the variation between the model and the real plant is represented in the predicted output by the forced response. 
This allows the control of the system when it is subject to an uncertain system.

To evaluate the performance of each controller, we consider controller characteristic parameters such as maximum overshoot, delay time, rise time, settling time, and a steady-state error.

Fig. 2 shows the step response of the suspension deflection, and we see that the overshoot percentage and settling time requirements are satisfied. The output has a settling time less than 1.2 seconds and an overshoot of less than $5 \%$. Moreover, the steady-state error approaches zero as well. Therefore, we determine that the response is satisfactory. It can be seen from Fig. 2 that better performance from the point of view of robustness and stability are obtained with RNPC. The RNPC can still stabilize the closed-loop system with no obvious degradation on performance. Using the proposed control approach, the fluctuations of suspension deflection is about $65 \%$ less than that of the open-loop and $45 \%$ less than that of the MPC control. We can also see that suspension deflection of the MPC controller presents more fluctuation. This impact produces a significant jerk on the car chassis and introduces undesired accelerations into the system and degrades the ride characteristics of the vehicle. But this situation does not happen in the RMPC.

In Fig. 3, we observe that the sprung mass acceleration of the RNPC controller yields the least value of the maximum sprung mass acceleration, compared with the MPC controller. In addition, we can see in Fig. 4 that the active control force constraint is respected by the RNPC control, while it is not respected by the MPC controller owing to its ignorance of the hard constraints in the controller design process. Consequently, the ride quality of the vehicle suspension system can be significantly improved, as compared with the standard MPC controller.

It can also be seen in Fig. 5 that the tire deflection of the RNPC is less than that of the MPC controller. This means that RNPC can produce better road holding, and it can be seen that the MPC improves ride and handling performance greatly.

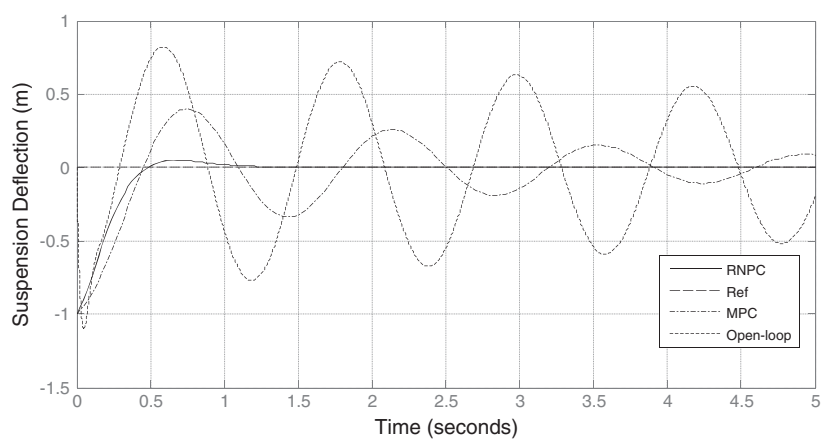

Fig. 2. Step response of suspension deflection.

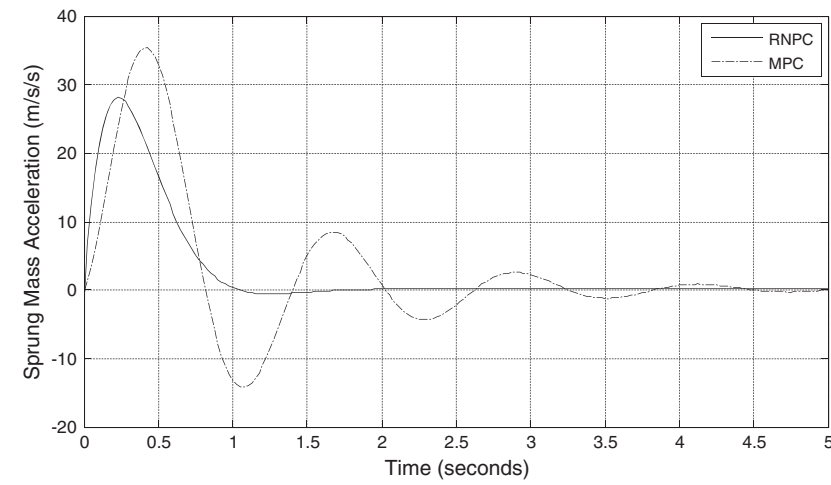

Fig. 3. Step response of sprung mass acceleration.

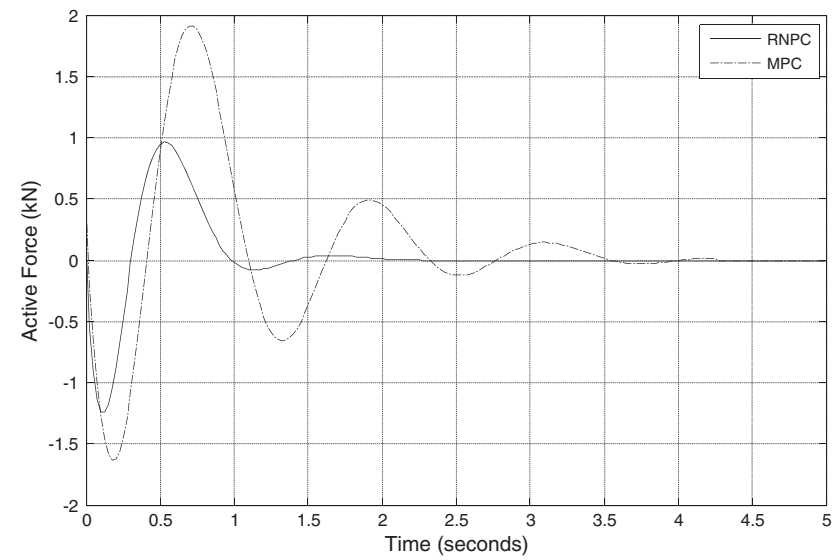

Fig. 4. Step response of active force.

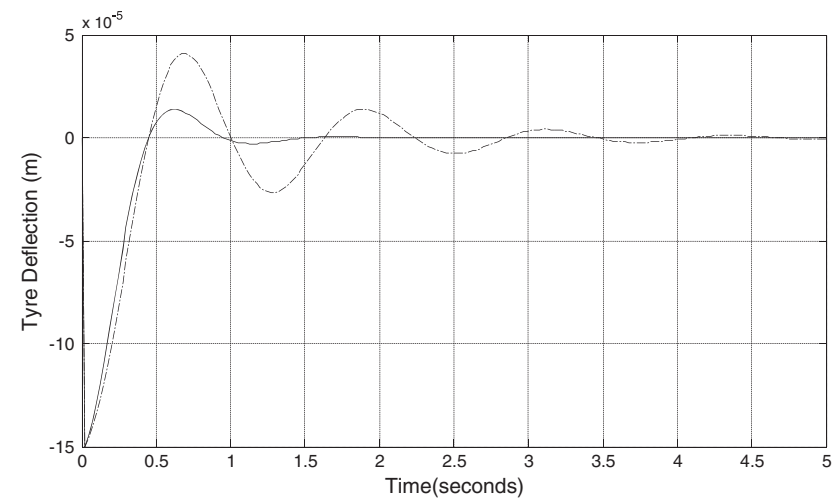

Fig. 5. Step response of tire deflection.

\section{CONCLUSION}

In this paper, the problem of robust fuzzy predictive control has been presented to design a controller for an active car suspension system under road disturbances. First, the sector nonlinearity method was used to construct the nonlinear active suspension system by T-S fuzzy system. 
An optimization method based on LMIs was introduced to reformulate the problem of non-linear quadratic programming to solve a robust model predictive control with constraints on both active suspension system and measured output, in such a way that road disturbances were considered as the output of the system. The terminal equality constraints were imposed and formulated in terms of LMIs to guarantee closed-loop stability. The control law provides the freedom to minimize vertical passenger acceleration, suspension travel, and tire deflections under road disturbances on the wheel. Future work will be focused on extending the RNPC for active faulttolerant control of vehicle active suspension systems.

\section{APPENDIX}

The design of a predictive controller demands the construction of a predictor. Then the construction of the predictor in the state-space representation can be conducted by iterating model (3) for more detail see [6]. In matrix representation the prediction can be seen as

$$
\hat{Y}=\Gamma+(\Lambda+\delta \Lambda) U_{n}
$$

After a mathematical manipulation of prediction, the matrices $\Lambda, \Gamma$ are given by

$$
\Lambda=\left[C_{n} \times H 0\right], \delta \Lambda=\left[\delta C_{n} \times \delta H 0\right]
$$

and

$$
\Gamma=C_{n} \Phi x(k)
$$

$$
\begin{aligned}
H & =\left[\begin{array}{cccc}
B & 0 & \ldots & 0 \\
A B & B & \ldots & 0 \\
\vdots & & & \\
A^{H p-2} B & A^{H p-3} B & \ldots & \sum_{i=0}^{H p-H u-1} A^{i} B \\
A^{H p-1} B & A^{H p-2} B & \ldots & \sum_{i=0}^{H p-H u} A^{i} B
\end{array}\right], \\
\delta H & =\left[\begin{array}{cccc}
\delta B & 0 & \ldots & 0 \\
\delta A \delta B & B & \ldots & 0 \\
\vdots & & & \\
\delta A^{H p-2} \delta B & \delta A^{H p-3} \delta B & \ldots & \sum_{i=0}^{H p-H u-1} \delta A^{i} \delta B \\
\delta A^{H p-1} \delta B & \delta A^{H p-2} \delta B & \ldots & \sum_{i=0}^{H p-H u} \delta A^{i} \delta B
\end{array}\right]
\end{aligned}
$$

$$
\begin{aligned}
\Phi & =\left[\begin{array}{l}
A \\
A^{2} \\
\vdots \\
A^{H p}
\end{array}\right], \quad C_{n}=\left[\begin{array}{cccc}
C & 0 & \ldots & 0 \\
0 & C & \ldots & 0 \\
\vdots & \vdots & \ddots & \vdots \\
0 & 0 & \ldots & C
\end{array}\right], \in R^{\text {Hpno } \times \text { Hpns }}, \\
\delta C_{n} & =\left[\begin{array}{cccc}
\delta C & 0 & \ldots & 0 \\
0 & \delta C & \ldots & 0 \\
\vdots & \vdots & \ddots & \vdots \\
0 & 0 & \ldots & \delta C
\end{array}\right], \in R^{\text {Hpno } \times \text { Hpns }}
\end{aligned}
$$

i. Saturation of the control signal

$$
\begin{gathered}
u_{\min }(k+j-1)<u(k+j-1)<u_{\max }(k+j-1) \\
, \quad j=1, \ldots, H_{u}+1
\end{gathered}
$$

This constraint can be grouped under the following matrix form

$$
U_{\min }<I U_{n}<U_{\max }
$$

where

$$
I_{H_{u}+1}=\left[\begin{array}{cccc}
1 & 0 & \ldots & 0 \\
0 & 1 & \ldots & 0 \\
\vdots & \vdots & \ddots & \vdots \\
0 & 0 & \ldots & 1
\end{array}\right], \in R^{H_{u}+1 \times H_{u}+1}
$$

(33) can be written in the matrix form as

$$
\left[\begin{array}{l}
I_{H_{u}+1} \\
-I_{H_{u}+1}
\end{array}\right] U_{n}<\left[\begin{array}{l}
U_{\max } \\
-U_{\min }
\end{array}\right]
$$

\section{ii. Saturation of the increment control}

$$
\begin{array}{r}
\Delta u_{\min }(k+j-1)<\sum_{i=1}^{j} u(k+j-1)-u(k-1) \\
<\Delta u_{\max }(k+j-1), \quad j=1, \ldots, H_{u}+1
\end{array}
$$

This gives in matrix form

$U_{\min }+U_{k-1}<L_{H_{u}+1} U_{n}<U_{\max }+U_{k-1}$ 


$$
\begin{aligned}
L_{H_{u}+1} & =\left[\begin{array}{cccc}
1 & 0 & \ldots & 0 \\
1 & 1 & \ldots & 0 \\
\vdots & \vdots & \ddots & \vdots \\
1 & 1 & \ldots & 1
\end{array}\right], \in R^{H_{u}+1 \times H_{u}+1}, \\
U_{k-1} & =\left[\begin{array}{l}
u(k-1) \\
u(k-1) \\
\vdots \\
u(k-1)
\end{array}\right], \in R^{(H u+1) n i}
\end{aligned}
$$

(36) can be written in the matrix form as

$$
\left[\begin{array}{c}
L_{H_{u}+1} \\
-L_{H_{u}+1}
\end{array}\right] U_{n}<\left[\begin{array}{l}
U_{\max }+U_{k-1} \\
-U_{\min }-U_{k-1}
\end{array}\right]
$$

\section{iii. Saturation of the output signal}

$$
y_{\min }<\hat{y}(k+j)<y_{\max }, j=1, \ldots, H_{p}
$$

Then inserting (29) into (38) yields

$$
Y_{\min }<\Gamma+(\Lambda+\delta \Lambda) U_{n}<Y_{\max }
$$

That can be transformed in the matrix inequalities

$$
\left[\begin{array}{l}
(\Lambda+\delta \Lambda) \\
-(\Lambda+\delta \Lambda)
\end{array}\right] U_{n}<\left[\begin{array}{c}
Y_{\max }-\Gamma \\
-Y_{\min }+\Gamma
\end{array}\right]
$$

\section{APPENDIX}

Similarly we calculate the predicted output of the horizon that represents the additional terminal constraints; here we assume that the system is still uncertain

$$
\hat{Y}_{c}=\Gamma_{c}+\left(\Lambda_{c}+\delta \Lambda_{c}\right) U_{n}
$$

After a mathematical manipulation of prediction, the matrices $\Lambda_{c}, \delta \Lambda_{c}, \Gamma_{c}$ are given by

$$
\Lambda_{c}=\left[C_{n c} \times H_{c} 0\right], \delta \Lambda_{c}=\left[\delta C_{n c} \times \delta H_{c} 0\right]
$$

and

$$
\Gamma_{c}=C_{n c} \Phi_{c} x(k)
$$

$$
\begin{aligned}
& H_{c}=\left[\begin{array}{cccc}
A^{H p} B & A^{H p-1} B & \cdots & \sum_{i=0}^{H p-H u+1} A^{i} B \\
A^{H p+1} B & A^{H p} B & \ldots & \sum_{i=0}^{H p-H u+2} A^{i} B \\
\vdots & \vdots & \ddots & \vdots \\
A^{H p+m-1} B & A^{H p+m-2} B & \ldots & \sum_{i=0}^{H p-H u+m} A^{i} B
\end{array}\right], \\
& H_{c}=\left[\begin{array}{cccc}
\delta A^{H p} \delta B & \delta A^{H p-1} \delta B & \ldots & \sum_{i=0}^{H p-H u+1} \delta A^{i} \delta B \\
\delta A^{H p+1} \delta B & \delta A^{H p} \delta B & \ldots & \sum_{i=0}^{H p-H u+2} \delta A^{i} \delta B \\
\vdots & \vdots & \ddots & \vdots \\
A^{H p+m-1} B & A^{H p+m-2} B & \ldots & \sum_{i=0}^{H p-H u+m} \delta A^{i} \delta B
\end{array}\right] \\
& \Phi=\left[\begin{array}{l}
A^{H p+1} \\
A^{H p+2} \\
\vdots \\
A^{H p+m}
\end{array}\right], C_{n}=\left[\begin{array}{cccc}
C & 0 & \ldots & 0 \\
0 & C & \ldots & 0 \\
\vdots & \vdots & \ddots & \vdots \\
0 & 0 & \ldots & C
\end{array}\right], \in R^{m n o \times m n s}
\end{aligned}
$$

where $n o, n i$, and $n s$ are the number of system outputs, system input, and system order, respectively.

This constraint leads to the following condition

$$
y\left(t+H_{p}+j\right)=y_{r e f}\left(t+H_{p}\right), j=1, \cdots, m
$$

Substituting (41) into (44) we obtain the following matrix form

$$
\left(\Lambda_{c}+\delta \Lambda_{c}\right) U_{n}=Y_{\text {refc }}-\Gamma_{c}
$$

(45) can be reformulated in terms of optimizing an upper bound, written in the following form

$$
\min \alpha \in R^{+}
$$

\section{Subject to}

$$
\left[\begin{array}{l}
\left(\Lambda_{c}+\delta \Lambda_{c}\right) \\
-\left(\Lambda_{c}+\delta \Lambda_{c}\right)
\end{array}\right] U_{n}<\left[\begin{array}{l}
Y_{r e f}-\Gamma_{c}+\alpha I_{m} \\
-Y_{r e f}+\Gamma_{c}+\alpha I_{m}
\end{array}\right]
$$

where $I_{m}$ is the identity matrix of dimension $m$.

\section{REFERENCES}

1. Göhrle, C., A. Schindler, A. Wagner, and O. Sawodny, "Design and vehicle implementation of preview active suspension controllers," IEEE Trans. Control Syst. Technol., Vol. 22, No. 3, pp. 1135-1142 (2014).

2. Crews, J. H., M. G. Mattson, and G. D. Buckner, "Multi-objective control optimization for semi-active vehicle suspensions," J. Sound Vibr., Vol. 330, No. 23, pp. 5502-5516 (2011).

3. Han, S. Y., G. Y. Tang, Y. H. Chen, X. X. Yang, and $\mathrm{X}$. Yang, "Optimal vibration control for vehicle active suspension discrete-time systems with actuator time delay," Asian J. Control, Vol. 15, No. 6, pp. 1579-1588 (2013). 


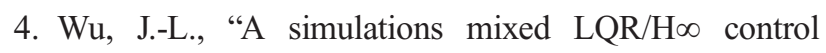
approach to the design of reliable active suspension controllers," Asian J. Control, (2015). DOI: 10.1002/ asjc. 1058.

5. Jahromi, A. F. and A. Zabihollah, "Linear quadratic regulator and fuzzy controller application in full-car model of suspension system with magnetor-heological shock absorber," in Proceedings of IEEE/ASME International Conference on Mechatronics and Embedded Systems and Applications, pp. 522-528 (2010).

6. Wong, P.-K., H. Shaojia, X. Tao, W. H. Cheong, and $\mathrm{X}$. Zhengchao, "Design of a new suspension system controlled by fuzzy-PID with wheelbase preview," Adv. Mech. Eng. II, Adv. Mech. Mater., Vol. 192, pp. 106-110 (2012).

7. Kim, C. and P. I. Ro, "A sliding mode controller for vehicle active suspension systems with non-linearities," Proc. Inst. Mech. Eng. Part D J. Automob. Eng., Vol. 212, No. 2, pp. 79-92 (1998).

8. Al-Holou, N., T. Lahdhiri, D. S. Joo, J. Weaver, and F. Al-Abbas, "Sliding mode neural network inference fuzzy logic control for active suspension systems," IEEE Trans. Fuzzy Syst., Vol. 10, No. 2, pp. 234-246 (2002).

9. Fialhm, I. and G. J. Balas, "Road adaptive active suspension design using linear parameter-varying gain-scheduling," IEEE Trans. Control Syst. Technol., Vol. 10, No. 1, pp. 43-54 (2002).

10. Demir, O., I. Keskin, and S. Cetin, "Modeling and control of a nonlinear half-vehicle suspension system: A hybrid fuzzy logic approach," Nonlinear Dyn., Vol. 67, pp. 2139-2151 (2012).

11. Anwar, S. "Generalized predictive control of yaw dynamics of a hybrid brake-by-wire equipped vehicle," Mechatronics, Vol. 15, pp. 1089-1108 (2005).

12. Shoukry, Y., M. W. El-Kharashi, and S. Hammad, "An embedded implementation of the Generalized Predictive Control algorithm applied to automotive active suspension systems," Comput. Electr. Eng., Vol. 39, No. 2, pp. 512-529 (2013).

13. Constantin, F., M. L. Caruntu, R. H. Gielen, P. P. J. van den Bosch, and S. Di Cairano, "Lyapunov based predictive control of vehicle drivetrains over CAN," Control Eng. Practice, Vol. 21, No. 12, pp. 1884-1898 (2013).

14. Bououden, S., M. Chadli, et al., "A new approach for fuzzy predictive adaptive controller design using particle swarm optimization algorithm," Int. J. Innovative Comput., Inf. Control, Vol. 9, No. 9, pp. 3741-3758 (2013).

15. Li, H., J. Yu, C. Hilton, and H. Liu, "Adaptive sliding mode control for nonlinear active suspension vehicle systems," IEEE Trans. Ind. Electron., Vol. 60, No. 8, pp. 3328-3338 (2013).
16. Li, H., X. Jing, H. K. Lam, and P. Shi, "Fuzzy sampleddata control for uncertain vehicle suspension systems," IEEE T. Cybern., Vol. 44, No. 7, pp. 1111-1126 (2014).

17. Orukpe, P. E., X. Zheng, I. M. Jaimoukha, A. C. Zolotas, and R. M. Goodall, "Model predictive control based on mixed $\mathrm{H} 2 / \mathrm{H} \infty$ control approach for active vibration control of railway vehicles," Veh. Syst. Dyn., Vol. 46, pp. 151-160 (2008).

18. Canale, M., M. Milanese, and C. Novara, "Semi-active suspension control using 'fast' model-predictive techniques," IEEE Trans. Control Syst. Technol., Vol. 14, No. 6, pp. 1034-1046 (2006).

19. Takagi, T., and M. Sugeno, "Fuzzy identification of systems and its application to modeling and control," IEEE Trans. Syst. Man. Cybern., Vol. 15, No. 1, pp. 116-132 (1985).

20. Ohtake, H., K. Tanaka, and H. O. Wang, "Fuzzy model-based servo and model following control for nonlinear systems," IEEE Trans. Syst. Man. Cybern. Part B-Cybern., Vol. 39, No. 6, pp. 1634-1639 (2009).

21. Chadli, M., and M. Darouach, "Robust admissibility of uncertain switched singular systems," Int. J. Control, Vol. 84, No. 10, pp. 1587-1600 (2011).

22. Aouaouda, S., M. Chadli, V. Cocquempot, and M. T. Khadir, "Multi-objective $\mathrm{H}-\mathrm{H} \infty$ faults detection observer design for Takagi-Sugeno fuzzy systems with unmeasurable premise variables: descriptor approach," Int. J. Adapt. Control Signal Process., Vol. 27, No. 12, pp. 1031-1047 (2013).

23. Espinosa, J., Vandewalle, J., and Wertz, V., Fuzzy Logic, Identification and Predictive Control, Springer, Berlin (2005).

24. Maciejowski, J., Predictive Control with Constraints, Prentice Hall, London (2002).

25. Bououden, B., M. Chadli, S. Filali, and A. El Hajjaji, "Fuzzy model based multivariable predictive control of a variable speed wind turbine: LMI approach," Renew. Energy, Vol. 37, No. 1. pp. 434-439 (2012).

26. Rawlings, J. B., and Mayne, D. Q., Model Predictive Control: Theory and Design, Nob Hill, Madison, WI (2009).

27. Saifia, D., M. Chadli, S. Labiod, and T. M. Guerra, "Robust $\mathrm{H} \infty$ static output feedback stabilization of T-S fuzzy systems subject to actuator saturation," Int. J. Control Autom. Syst., Vol. 10, No. 3, pp. 613-622 (2012).

28. Bazaraa, M. S., Sherali, H. D., and Shetty, C. M., Nonlinear Programming. Theory and Algorithms, 3rd edn., John Wiley \& Sons, Hoboken (2006).

29. Fletcher, R. "Minimizing general functions subject to linear constraints, Numerical methods for non-linear optimization," F. A. Lootsma (ed.), pp. 279-296, Academic Press, London (1972). 
30. Fletcher, R., Practical Methods of Optimization, 2nd edn., John Wiley \& Sons, New York (2000).

31. Gill, P. E., and W. Murray, "Linearly constrained problems including linear and quadratic programming," in D. Jacobs (ed.), The State of the Art in Numerical Analysis, Academic Press, London and New York, pp. 313-363 (1977).

32. Boyd, S., El Ghaoui, L., Feron, E., and Balakrishnan, V., Linear Matrix Inequalities In System and Control Theory, SIAM, Philadelphia, USA (1994). 